\title{
Matrix metalloproteinase 13 genotype in rs640198 polymorphism is associated with severe coronary artery disease
}

\author{
Anna Vaškư ${ }^{\mathrm{a}, *}$, Jaroslav Meluzín ${ }^{\mathrm{b}}, \mathrm{Jan}_{\text {Bahák }}^{\mathrm{a}}$, Vladimír Kincl ${ }^{\mathrm{b}}$, Monika Pávková Goldbergováa \\ Jan Sitar ${ }^{\mathrm{b}}$, Filip Zlámal ${ }^{\mathrm{a}}$, Julie Bienertová-Vaškü ${ }^{\mathrm{a}}$ and Jiř́ Vítovec ${ }^{\mathrm{b}}$ \\ ${ }^{a}$ Department of Pathological Physiology, Faculty of Medicine, Masaryk University, Brno, Czech Republic \\ ${ }^{\mathrm{b}} 1$ st. Department of Internal Medicine/Cardioangiology, ICRC, St. Anna Hospital, Masaryk University, Brno, \\ Czech Republic
}

\begin{abstract}
Atherosclerosis as a main etiopathogenetic source for coronary artery disease (CAD) development is intimately related to dynamic changes in the extracellular matrix (ECM). Elevated levels of MMP-13 have been observed in human atherosclerotic plaques which could also involve variability in MMP-13 gene. The aim of the study was to associate rs640198 polymorphism with CAD and/or with its severity.

The study comprised 1071 consecutive patients with suspected or known coronary artery disease (CAD), confirmed by coronary angiography.

Genotyping for the rs640198 polymorphism in MMP-13 gene was performed using Taqman ${ }^{\circledR}$ assay. The TT and TG genotypes of rs640198 polymorphism in MMP-13 gene confer the significantly increased risk of triple vessel disease compared to patients without atherosclerotic lesions in coronary arteries (odds ratio $=1.64$, Pcorr $=0.05$ ). Furthermore, an increased risk of having 5 and more stenoses (odds ratio $=1.90$, Pcorr $=0.004$ ) was observed in TT and TG carriers (sensitivity of 0.613 and a specificity of 0.544 ; power of the test is 0.87 ).

The T allele of MMP-13 intron polymorphism rs640198 is associated with the severity of coronary artery disease, represented by the number of affected arteries as well as by the number of stenoses confirmed by coronarography.
\end{abstract}

Keywords: MMP-13, CAD, rs640198, severity

\section{Introduction}

The cardiovascular diseases, especially the coronary artery disease (CAD), represent one of the most common causes of mortality in developed countries. The genetic background that underlies the susceptibility, occurrence and severity of CAD, however, is still poorly elucidated [10].

Atherosclerotic process as a base for CAD onset is intimately related to dynamic changes in the extracellular matrix (ECM) and its associated proteins: the re-

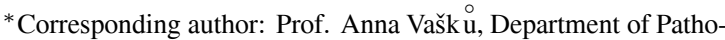
logical Physiology, Faculty of Medicine, Masaryk University Brno, University Campus Bohunice, A18, Kamenice 5, 62500 Brno, Czech Republic. Tel.: +420 549498 299; Fax: +420 549494 340; E-mail: avasku@med.muni.cz. tention of lipoproteins by proteoglycans leads to the development of atherosclerotic lesions, but ultimately, it is the breakdown of ECM that facilitates plaque ruptures, triggering the onset of clinical symptoms of CAD [8]. The stability of the plaque depends on its collagen content; degradation of the collagen leads to a vulnerable plaque that is prone to rupture [4]. Therefore, matrix metalloproteinases (MMPs) which are able to cleave collagens should play an important role in the pathogenesis of atherosclerosis, plaque rupture and aneurysm [16,21]. The expression of various MMPs, along with their endogenous inhibitors, seems to be differentially regulated dependently on the lesion progression [30].

MMP-13 is active against types I and III collagens $[11,18]$. MMP-13 can be further distinguished from other interstitial collagenases by its ability to 
cleave gelatin, proteoglycans, tenascin, fibronectin, as well as its capacity to activate latent proMMPs [13]. It seems to have a unique role in the accelerated collagen turnover associated with various conditions [18].

The intimal macrophages seem to be the major source of MMP-13 production [27,30]. MMP-13 colocalized with MMP-1 in human atheroma is localized mostly in the shoulder region of the plaques as well as in areas surrounding the lipid core. Levels of MMP-1 as well as MMP-13 in atheromatous plaques exceed those in fibrous plaques, corresponding to the content of macrophages [27]. The increased expression of MMPs in the atheroma also leads to increased MMP levels in the circulation [4].

Late AT II (angiotensin II) treatment was reported to augment lesion size of fully developed atherosclerotic plaques with macrophage-rich phenotype in ApoE null mice atherosclerotic model [6]. Amplified collagen type I degradation in this case is related to increase of active MMP- 8 and MMP-13 levels in the macrophagerich plaque [6].

However, MMP-13 was also proposed as another possible candidate for the NO-mediated prevention of atherosclerosis in the model of atherosclerotic NOS3/apoE-null mice: MMP-13 is able to cleave ICAM-1, both in vivo and in vitro [28]. Increased levels of soluble ICAM-1 (together with VCAM-1, E-selectin and P-selectin) as markers of inflammation were associated with the development of atherosclerosis and clinical expression of CAD [16]. An antiatherogenic effect of MMP-13 is further documented by its ability to process human prolactin in vitro to a $16-\mathrm{kDa}$ fragment with strong antiangiogenic properties [17] and by degrading MCP-3 which is functioning as a potent proinflammatory stimulus in atherosclerotic lesions [19].

MMP13 gene is encoded by a 10.5 kilobase gene located on human chromosome 11q22.2-q22.3 [21]. Sequence analysis of the 5' - flanking region of the gene revealed the presence of a TATA box, an AP-1 motif, a PEA-3 consensus motif, an osteoblast specific element (OSE-2) and a TGF- $\beta$ inhibitory element [20,22].

MMP-13 expression was observed to be nearly absent in the normal arterial wall, but was found to be remarkably increased with atherosclerotic lesion progression $[27,30]$ which could be caused by variability of MMP-13 gene. So far, six polymorphisms in MMP-13 gene were identified (ALFRED, http://alfred.med.yale. edu/alfred/recordinfo.asp?condition=sites.site_uid=

'SI001554P). The intron rs640198 polymorphism was chosen for sufficiently high frequency of the polymorphism in European population (ALFRED). Further- more, in case of rs640198, the important difference can be found in transcription profiles of both alleles of the polymorphism in silico: the transcription factor Pbx 1 factor can bind to the allele $\mathrm{G}$, not to the allele $\mathrm{T}$. It is known that the homeodomain transcription factor $\mathrm{Pbx} 1$ orchestrates separate transcriptional pathways to control great-artery patterning and cardiac outflow tract septation in mice [5]. Pbx1-null embryos display anomalous great arteries owing to a failure to establish the initial complement of branchial arch arteries in the caudal pharyngeal region. Pbx1 deficiency also results in the failure of cardiac outflow tract septation [5]. Interactions between alleles of $3 \mathrm{Pbx}$ genes, which encoded homeodomain transcription factors, were shown to be sufficient to determine the phenotypic presentation of congenital heart diseases in mice [26]. From this point of view, absence of $\mathrm{Pbx} 1$ binding sequence in the T allele of MMP-13 gene can modify the collagen cleaving activity of MMP-13 during development and thus, to take part in suboptimal development of cardiovascular system including coronary artery network in the $\mathrm{T}$ allele carriers.

The aim of the study was to associate rs640198 polymorphism with CAD and/or its severity and also with congenital vascular abnormalities in the CentralEuropean patients with CAD.

\section{Materials and methods}

\subsection{Experimental subjects}

The study comprised 1071 consecutive patients of Central-European, Caucasian origin with suspected or known CAD referred to the 1st Department of Internal Medicine/ Cardioangiology for coronary angiography between October 2005 and February 2007. During the short-term hospitalization the patients underwent full cardiologic investigation (history, physical examination, electrocardiography, laboratory examination, coronary angiography, echocardiography in patients with unclear diagnosis). The study was approved by the institutional ethics committee; informed consent of all patients is archived.

Coronary angiography was performed using the standard technique. The coronary lesions were visually analyzed in multiple projections. Based on morphology of coronary arteries, 2 patient groups were defined: 1, patients with significant coronary atherosclerosis (at least one coronary artery with luminal diameter narrowing of $50 \%$ or more, CAD group) and 2, patients with 
Table 1

Descriptive statistics of major variables in different MMP-13 genotypes

\begin{tabular}{|c|c|c|c|c|}
\hline & $\begin{array}{c}\text { MMP-13 GG } \\
(N=521)\end{array}$ & $\begin{array}{c}\text { MMP-13 GT } \\
(N=459)\end{array}$ & $\begin{array}{c}\text { MMP-13 TT } \\
(N=91)\end{array}$ & $\mathrm{P}$-value \\
\hline Age (median, range) & $41(12-94)$ & $41(18-104)$ & $40(18-61)$ & 0.582 \\
\hline $\operatorname{Sex}(\mathrm{M} / \mathrm{F}, \%$ of men) & $360(69 \%)$ & $309(67 \%)$ & $65(71 \%)$ & $\begin{array}{l}\mathrm{Pg}=0.689 \\
\mathrm{~Pa}=0.975\end{array}$ \\
\hline Hyperlipidemia & $325(62 \%)$ & $270(59 \%)$ & $57(63 \%)$ & $\begin{array}{l}\mathrm{Pg}=0.490 \\
\mathrm{~Pa}=0.547\end{array}$ \\
\hline Hypertension & $418(80 \%)$ & $357(78 \%)$ & $75(82 \%)$ & $\begin{array}{l}\mathrm{Pg}=0.481 \\
\mathrm{~Pa}=0.840\end{array}$ \\
\hline Diabetes mellitus & $154(30 \%)$ & $143(31 \%)$ & $30(33 \%)$ & $\begin{array}{l}\mathrm{Pg}=0.752 \\
\mathrm{~Pa}=0.455\end{array}$ \\
\hline Obesity & $172(33 \%)$ & $123(27 \%)$ & $31(34 \%)$ & $\begin{array}{l}\mathrm{Pg}=0.08 \\
\mathrm{~Pa}=0.230\end{array}$ \\
\hline Smoking & $66(13 \%)$ & $65(14 \%)$ & $19(21 \%)$ & $\begin{array}{l}\mathrm{Pg}=0.113 \\
\mathrm{~Pa}=0.07\end{array}$ \\
\hline $\begin{array}{l}\text { Family history of CAD } \\
\text { or stroke before age of } \\
60 \text { years }(N=868)\end{array}$ & $98(N=420,23 \%)$ & $68(N=373,18 \%)$ & $14(N=75,19 \%)$ & $\begin{array}{l}\mathrm{Pg}=0.188 \\
\mathrm{~Pa}=0.108\end{array}$ \\
\hline CAD & $398(76 \%)$ & $368(80 \%)$ & $79(87 \%)$ & $\begin{array}{l}\mathrm{Pa}=0.02 \\
\mathrm{Pg}=0.06\end{array}$ \\
\hline
\end{tabular}

$\mathrm{CAD}=$ coronary artery disease.

Pg: probability of a difference in genotype distribution.

Pa: probability of a difference in allelic frequencies.

insignificant coronary atherosclerosis (stenoses with luminal diameter narrowing below $50 \%$ or luminal irregularities), and patients with normal smooth coronary arteries. In addition, the absolute number of significant coronary artery stenoses (irrespective of the vessels involved) was calculated.

Another 203 healthy individuals with negative cardiovascular disease history were recruited from general practitioner office.

\subsection{Analysis of MMP-13 polymorphism (rs640198)}

To analyse polymorphism, blood samples were drawn and stored at a temperature below $-18^{\circ} \mathrm{C}$. The DNA was extracted from periphery blood leukocytes using proteinase $\mathrm{K}$ and precipitated by isopropanole and chloroform. DNA was extracted from peripheral leukocytes by using the standard proteinase $\mathrm{K}$ method. MMP-13 gene polymorphism rs640198, T/G in position 6373 , on the chromosome 11 was detected in the study.

Genotyping of rs640198 polymorphism in MMP-13 gene was performed using 5'exonuclease (Taqman ${ }^{\circledR}$ ) chemistry on the ABI Prism ${ }^{\circledR} 7000$ (Applied Biosystems, Foster City, CA). All assays were validated by direct sequencing using Big Dye v 1.1 terminator chemistry (Applied Biosystems).

\subsection{Analysis of MMP-13 plasma levels}

Plasma concentrations of MMP-13 were determined by ELISA in 20 healthy individuals.

\subsection{Statistical analysis}

Continuous variables are given as a median and range; categorical variables are presented as numbers (percentage). For analysis of differences among the groups, non-parametric test (Mann-Whitney U test, Kruskal-Wallis ANOVA test) or Fisher's exact test were chosen. The distributions of genotype and allelic frequencies and their differences were calculated using chi-square test. The Bonferroni correction (Pcorr) for multiple comparisons was used when necessary.

Linear regression model was used to create multivariate model of relationships among MMP-13 genotype (GG+GT vs. TT), age, sex, HDL, CRP and statins therapy.

Statistical package Statistica, Version 9 was used for analyses. A $p<0.05$ was considered significant.

\section{Results}

Basic description of the study cohort according to the genotyped of investigated polymorphism is given in Table 1 , we found a marginally significant differ- 
Table 2

Number of affected arteries and MMP-13 genotypes

\begin{tabular}{|c|c|c|c|c|c|c|c|c|}
\hline No of affected arteries & MMP-13-TT & MMP-13-TG & MMP-13-GG & $\begin{array}{c}\text { Frequency of } \\
\text { alleles } \mathrm{T} / \mathrm{G}\end{array}$ & $\mathrm{Pg}$ & $\mathrm{Pa}$ & Pg-corr & Pa-corr \\
\hline CAD1 $(N=252)$ & $24(10 \%)$ & $101(40 \%)$ & $127(50 \%)$ & $0.296 / 0.704$ & 0.104 & 0.125 & & \\
\hline CAD2 $(N=252)$ & $20(8 \%)$ & $108(43 \%)$ & $124(49 \%)$ & $0.294 / 0.706$ & 0.199 & 0.139 & & \\
\hline CAD3 $(N=272)$ & $29(11 \%)$ & $127(46 \%)$ & $116(43 \%)$ & $0.340 / 0.660$ & 0.01 & 0.006 & 0.05 & 0.03 \\
\hline CAD4 $(N=69)$ & $6(9 \%)$ & $32(46 \%)$ & $31(45 \%)$ & $0.319 / 0.681$ & 0.176 & 0.104 & & \\
\hline Non CAD5 $(N=106)$ & $8(8 \%)$ & $41(39 \%)$ & $57(53 \%)$ & $0.269 / 0.731$ & 0.364 & 0.508 & & \\
\hline Non CAD6 $(N=120)$ & $4(3 \%)$ & $50(42 \%)$ & $66(55 \%)$ & $0.242 / 0.758$ & & & & \\
\hline All groups $(N=1071)$ & $91(8 \%)$ & $459(43 \%)$ & $521(49 \%)$ & $0.300 / 0.700$ & & & & \\
\hline Healthy control group $(N=203)$ & $21(10 \%)$ & $79(39 \%)$ & $103(51 \%)$ & $0.298 / 0.702$ & 0.07 & 0.122 & & \\
\hline
\end{tabular}

CAD1 - coronary artery diseases with one affected vessel.

CAD2 - coronary artery disease with two affected vessels.

CAD3 - with three affected vessels.

CAD4 - with truncal affection.

Non-CAD5 - without significant stenosis.

Non-CAD6 - only smooth arteries.

Pg: probability of a difference in genotype distribution.

Pa: probability of a difference in allelic frequencies.

Pgcorr, Pacorr: P-values corrected for multiple comparison.

ence in MMP-13 polymorphisms between patients with clinically manifest $\mathrm{CAD}$ compared to those without the disease $(\mathrm{Pg}=0.06, \mathrm{~Pa}=0.02$, Table 1$)$.

When CAD group had been further stratified according to the number of affected arteries, significant differences in MMP-13 genotype distribution as well as in allelic frequency between patients with triple vessel disease (CAD 3) and persons without affection of coronary arteries (Non-CAD 6) were observed (Pgcorr = 0.05 , Pacorr $=0.03$, Table 2 ). In codominant model of heredity, the TT and TG genotypes are associated with significantly higher risk of triple vessel disease compared to patients without atherosclerotic lesions (odds ratio $=1.64,95 \%$ confidential interval 1.07-2.53, Pcorr $=0.05$, sensitivity 0.57 , specificity 0.55 , power test 0.62). No significant differences were observed between healthy individuals and patients without affections of coronary arteries (Table 2).

When the number of significant stenoses had been associated with MMP-13, significant differences in genotypes distributions as well as in allelic frequencies between patients with 5 and more stenoses compared to the patients without atherosclerotic lesions were found $($ Pgcorr $=0.04$, Pacorr $=0.02$, respectively, Table 3). Also, an increase risk of carrying five and more stenoses (odds ratio $=1.90,95 \%$ confidential interval 1.26-2.86, Pcorr $=0.004)$ was observed in TT and TG carriers. This potential marker has a sensitivity of 0.61 and a specificity of 0.54 ; the power test is 0.87 .

When linear regression model was used to create multivariate model of relationships among MMP-13 genotype (GG+GT vs. TT), age, sex, HDL, CRP and statins therapy, the MMP-13 genotype was not found to be a significant independent predictor of CAD (Table 4).

Furthermore, we evaluated the prevalence of congenital vascular abnormalities and their association with rs640198 polymorphism in our group of patients. Although aortic aneurysm was identified only in 7 patients $(=0.7 \%)$, the increased frequency of the $\mathrm{T}$ allele was observed in this small group $(\mathrm{Pa}=0.03$, data not shown). The GT genotype was almost four times more frequent in $23(2 \%)$ patients with pulmonary embolism $(\mathrm{OR}=3.88$, Pcorr $=0.007)$ compared to $\mathrm{CAD}$ patients without pulmonary embolism with sensitivity 0.74 , specificity 0.59 ; power test was 0.84 (data not shown).

The MMP-13 plasma levels were evaluated in 20 healthy individuals: only very low levels of the matrix metalloproteinase-13 were detected $(0.021 \pm$ $0.01 \mathrm{ng} / \mathrm{mL})$. No significant differences among MMP13 genotypes were observed in the group $(P=0.571)$.

\section{Discussion}

In our previous study, two different haplotypes in promoter of MMP-2 gene were observed to be significantly more or less frequent in persons with coronary triple vessel disease compared to non-ischemic persons [29]. In the present study, the rs640198 (intronic) polymorphism in MMP-13 gene was associated with triple vessel disease and also with presence of five and more stenoses of coronary arteries. Functional consequence between MMP-2 and MMP-13 can be considered, as MMP-2 is able to activate MMP-13s from 
Table 3

Number of significant stenoses and MMP-13 genotypes

\begin{tabular}{|c|c|c|c|c|c|c|c|c|}
\hline No of significant stenoses & MMP-13-TT & MMP-13-TG & MMP-13-GG & $\begin{array}{c}\text { Frequency of } \\
\text { alleles T/G }\end{array}$ & $\mathrm{Pg}$ & $\mathrm{Pa}$ & Pg-corr & Pa-corr \\
\hline$\geqslant 5(N=163)$ & $14(9 \%)$ & $86(53 \%)$ & $63(38 \%)$ & $0.350 / 0.750$ & 0.008 & 0.004 & 0.04 & 0.02 \\
\hline $4(N=123)$ & $10(8 \%)$ & $54(44 \%)$ & $59(48 \%)$ & $0.301 / 0.699$ & 0.308 & 0.188 & & \\
\hline $3(N=187)$ & $22(12 \%)$ & $67(36 \%)$ & $98(52 \%)$ & $0.297 / 0.703$ & 0.06 & 0.174 & & \\
\hline $2(N=167)$ & $12(7 \%)$ & $76(46 \%)$ & $79(47 \%)$ & $0.299 / 0.701$ & 0.346 & 0.162 & & \\
\hline $1(N=205)$ & $21(10 \%)$ & $85(42 \%)$ & $99(48 \%)$ & $0.310 / 0.690$ & 0.120 & 0.07 & & \\
\hline
\end{tabular}

Pg: probability of a difference in genotype distribution.

Pa: probability of a difference in allelic frequencies.

Pgcorr, Pacorr: P-values corrected for multiple comparison.

Table 4

Multiple logistic regression analysis for the association between MMP-13 rs640198 genotypes and susceptibility to CAD adjusting for confounding factors

\begin{tabular}{lcr}
\hline & Adjusted OR (95\% CI) & P -value \\
\hline MMP-13 & $1.34(0.76-2.38)$ & 0.31 \\
T allele & & \\
carriage & & \\
(TT and GT) & $0.19(0.10-0.37)$ & $<0.001$ \\
Sex (women vs. men) & $1.06(1.04-1.09)$ & $<0.001$ \\
Age & $0.26(0.12-0.56)$ & $<0.001$ \\
HDL & $1.03(1-1.06)$ & 0.006 \\
CRP & $57.78(31.97-104.43)$ & $<0.001$ \\
Statins (yes/no) & \multicolumn{2}{c}{} \\
\hline
\end{tabular}

HDL - high density lipoproteins.

$\mathrm{CRP}$ - C-reactive protein.

proMMP-13 and coordinate their proteolytic activities on extracellular matrix [3]. It has been reported previously that the structural remodelling of vessel wall during atherosclerotic process (elastin: collagen) is dictated by total MMP:TIMP ratio; this relation was shown to be substantially affected by blood flow in experimental study on adult male Sprague-Dawley rats [2]. Based on the results of this study, it can be concluded that not only MMPs expression or the blood levels of MMPs and their inhibitors, but also blood flow characteristics must be considered when vascular remodelling during atherosclerosis is evaluated [2].

Another aspect of great importance could be the prenatal role of the MMP-13 during organogenesis of cardiovascular system [1]. Recently, the MMP-13 (RNA as well as protein) was observed to be significantly downregulated during the second and the third trimester of pregnancy with respect to diversity and amount of matrix metalloproteinases produced and secreted by human decidua and invasive trophoblast [1]. This could have crucial effects on embryogenic development of cardiovascular system, as MMP13 may be involved in early stages of cardiovascular development [1]. The supposed decreased function of the T allele of rs640198
MMP-13 polymorphism associated with the loss of $\mathrm{Pbx}-1$ transcription factor site [5] could be related to suboptimal MMP-13 expression and therefore could play a role in early stages of heart development.

Based on this hypothesis and also on results of the presented study, another question arises as for possible consequence of CAD with some congenital heart malformations, even though below clinical significance. Recently, when the multidetector computed tomography (MDCT) angiography was used for CAD screening in a large general cardiology cohort $(N=4,543)$, other clinically relevant nonatherosclerotic cardiovascular diseases were visualized and identified unexpectedly [14] in $4.4 \%$ of investigated subjects $(N=$ 200). Most common abnormalities were: congenital coronary artery anomalies (38\%; largely right coronary artery from the left aortic sinus); ascending aortic aneurysms $>$ or $=45 \mathrm{~mm}(22 \%)$; hypertrophic cardiomyopathy with apical left ventricular (LV) wall thickening (14\%); valvular heart diseases (8\%), congenital heart diseases, including ventricular septal defect $(6 \%)$; pulmonary embolus $(6 \%)$; as well as LV noncompaction, left atrial myxoma, and LV apical aneurysm ( $2 \%$ each).

In our study, the $\mathrm{T}$ allele of the investigated polymorphism in MMP13 gene was more frequent in patients with aortic aneurysma and pulmonary embolism. This is in agreement with the recent findings that polymorphisms in MMP2, MMP3, MMP-13, and ELN genes may independently contribute to the pathogenesis of abdominal aortic aneurysma [23,24]. The structural changes in the venous wall in addition to the increased expression of MMP-2, MMP-9 and MMP-13 in venous aneurysm with pulmonary embolism compared to normal saphenous vein and varicous vein suggest a possible causal role for these MMPs in pathogenesis of these states [12]. Even though there is no evidence of the functional impact of the investigated polymorphism, 
based on the results of the presented studies, we believe that these findings support functional, not only statistical association of rs640198 MMP-13 polymorphism with coronary artery disease and pulmonary embolism and aortic aneurysm, observed in our study.

Moreover, other polymorphisms in other MMP genes, especially in MMP-3 and MMP-9 gene, have been previously reported to associate with the manifestation of coronary atherosclerosis and/or its progression, coronary restenosis development after therapy and plaques instability responsible for myocardium infarction development [7,9,25,31]. Certain genotypes of MMP-1, MMP-3 and MMP-9 with lower transcriptional activity were also associated with higher frequencies of arteriovenous fistula (AVF) failure in hemodialysis patients which may result from more accumulation of extracellular matrix, leading to AVF stenosis [15].

No information about MMP-13 levels in CAD have been reported so far in humans. However, MMP-2 as well as MMP-13 expression was found to be different in aorta, carotid artery, femoral artery and vena cava in animal model [2] which seemed to decrease the potential predictive value of MMP-13 blood levels. In a nother study, plasma levels of MMP-8 were associated with CAD [11].

Recently, a comprehensive analysis of extracellular matrix components comprising the vascular proteome in humans was performed [8]. The proteomics method allowed for the identification of scarce proteins present in the vascular extracellular space, identification of novel glycoproteins, and interrogation of proteolytic activity within tissues based on the identification of proteolytic enzymes and corresponding degradation products. These facts, if comprehensively characterized, could lead to development of the new therapeutic options for cardiovascular diseases [8].

In conclusion, the $\mathrm{T}$ allele of rs640198 polymorphism in MMP-13 gene was in our study associated with the severity of coronary artery disease characte-rized by the number of affected arteries as well as by the number of confirmed stenoses. More research into the pathophysiological role of MMP13 in early development of cardiovascular system as well as in pathophysiology of coronary artery disease and other adverse cardiovascular conditions is necessary.

\section{Acknowledgement}

The study was supported by a grant of the Internal Grant Agency of the Czech Republic IGA NS/10206$3 / 2009$.

\section{References}

[1] J. Anacker, S.E. Segerer, C. Hagemann, S. Feix, M. Kapp, R. Bausch, U. Kämmerer, Human decidua and invasive trophoblasts are rich sources of nearly all human matrix metalloproteinases. Mol Hum Reprod (12 May 2011). [Epub ahead of print].

[2] P. Basu, U. Sen, N. Tyagi, S.C. Tyagi, Blood flow interplays with elastin: collagen and MMP: TIMP ratios to maintain healthy vascular structure and function. Vasc Health Risk Manag 6 (2010), 215-28.

[3] J.L. Beaudeux, P. Giral, E. Bruckert, M.J. Foglietti, M.J. Chapman, [Matrix metalloproteinases and atherosclerosis. Therapeutic aspects]. Ann Biol Clin (Paris) 61 (2003), 147-58.

[4] C. Cevik, M. Otahbachi, K. Nugent, C. Warangkana, G. Meyerrose, Effect of 3-hydroxy-3-methylglutaryl coenzyme A reductase inhibition on serum matrix metalloproteinase-13 and tissue inhibitor matrix metalloproteinase-1 levels as a sign of plaque stabilization. J Cardiovasc Med (Hagerstown) 9 (2008), 1274-1278.

[5] C.P. Chang, K. Stankunas, C. Shang, S.C. Kao, K.Y. Twu, M.L. Cleary, $\mathrm{Pbx} 1$ functions in distinct regulatory networks to pattern the great arteries and cardiac outflow tract. Development 135 (2008), 3577-3586.

[6] C. Cheng, D. Tempel, R. van Haperen, L. van Damme, M. Algür, R. Krams, R. de Crom, Activation of MMP8 and MMP13 by angiotensin II correlates to severe intra-plaque hemorrhages and collagen breakdown in atherosclerotic lesions with a vulnerable phenotype. Atherosclerosis 204 (2009), 26-33.

[7] M.P. de Maat, J.W. Jukema, S. Ye, A.H. Zwinderman, P.H. Moghaddam, M. Beekman, J.J. Kastelein, A.J. van Boven, A.V. Bruschke, S.E. Humphries, C. Kluft, A.M. Henney, Effect of the stromelysin-1 promoter on efficacy of pravastatin in coronary atherosclerosis and restenosis. Am J Cardiol $\mathbf{8 3}$ (1999), 852-856.

[8] A. Didangelos, X. Yin, K. Mandal, M. Baumert, M. Jahangiri, M. Mayr, Proteomics characterization of extracellular space components in the human aorta, Mol Cell Proteomics 9 (2010), 2048-2062.

[9] S. Fallah, M. Seifi, A. Ghasemi, M. Firoozrai, A. Samadikuchaksaraei, Matrix metalloproteinase-9 and paraoxonase 1 Q/R192 gene polymorphisms and the risk of coronary artery stenosis in Iranian subjects. J Clin Lab Anal 24 (2010), 305310 .

[10] A.D. Hingorani, T. Shah, M. Kumari, R. Sofat, L. Smeeth, Translating genomics into improved healthcare, BMJ 341 (2010), c5945. doi: 10.1136/bmj.c5945.

[11] R. Kato, Y. Momiyama, R. Ohmori, H. Taniguchi, H. Nakamura, F. Ohsuzu, Plasma matrix metalloproteinase- 8 concentrations are associated with the presence and severity of coronary artery disease. Circ J 69 (2005), 1035-1040.

[12] C. Irwin, A. Synn, i. Kraiss, O. Zhang, M.M. Griffen, G.C. Hunter, Metalloproteinase expression in venous aneurysms. $J$ Vasc Surg 48 (2008), 1278-1285.

[13] V. Knäuper, H. Will, C. López-Otin, B. Smith, S.J. Atkinson, H. Stanton, R.M. Hembry, G. Murphy, Cellular mechanisms for human procollagenase-3 (MMP-13) activation. Evidence that MT1-MMP (MMP-14) and gelatinase a (MMP-2) are able to generate active enzyme. J Biol Chem 271 (1996), 1712417131.

[14] T. Knickelbine, J.R. Lesser, T.S. Haas, E.R. Brandenburg, B.K. Gleason-Han, B. Flygenring, T.F. Longe, R.S. Schwartz, B.J. Maron, Identification of unexpected nonatherosclerotic 
cardiovascular disease with coronary CT angiography. JACC Cardiovasc Imaging 2 (2009), 1085-1092.

[15] C.C. Lin, W.C. Yang, M.Y. Chung, P.C. Lee, Functional polymorphisms in matrix metalloproteinases-1, -3, -9 are associated with arteriovenous fistula patency in hemodialysis patients. Clin J Am Soc Nephrol 5 (2010), 1805-1814.

[16] H.H. Lu, Z.Q. Sheng, Y. Wang, L. Zhang, Levels of soluble adhesion molecules in patients with various clinical presentations of coronary atherosclerosis. Chin Med J (Engl) 123 (2010), 3123-3126

[17] Y. Macotela, M.B. Aguilar, J. Guzmán-Morales, J.C. Rivera, C. Zermeño, F. López-Barrera, G. Nava, C. Lavalle, G. Martínez de la Escalera, C. Clapp, Matrix metalloproteases from chondrocytes generate an antiangiogenic $16 \mathrm{kDa}$ prolactin. J Cell Sci 119 (2006), 1790-1800.

[18] D. Mao, J.K. Lee, S.J. VanVickle, R.W. Thompson, Expression of collagenase-3 (MMP-13) in human abdominal aortic aneurysms and vascular smooth muscle cells in culture. Biochem Biophys Res Commun 261 (1999), 904-910.

[19] G.A. McQuibban, J.H.Gong, J.P. Wong, J.L.Wallace, I. ClarkLewis, C.M. Overall, Matrix metalloproteinase processing of monocyte chemoattractant proteins generates $\mathrm{CC}$ chemokine receptor antagonists with anti-inflammatory properties in vivo. Blood 100 (2002), 1160-1167.

[20] P.G. Mitchell, H.A. Magna, L.M. Reeves, L.L. LoprestiMorrow, S.A.Yocum, P.J. Rosner, K.F. Geoghegan, J.E. Hambor, Cloning, expression, and type II collagenolytic activity of matrix metalloproteinase-13 from human osteoarthritic cartilage. J Clin Invest 97 (1996), 761-768.

[21] A.M. Pendás, I. Santamaría, M.V. Alvarez, M. Pritchard, C. López-Otín, Fine physical mapping of the human matrix metalloproteinase genes clustered on chromosome 11q22.3. Genomics 37 (1996), 266-268.

[22] A.M. Pendás, M. Balbín, E. Llano, M.G. Jiménez, C. LópezOtín, Structural analysis and promoter characterization of the human collagenase-3 gene (MMP13). Genomics 40 (1997), 222-33.

[23] K.D. Rizas, N. Ippagunta, M.D. 3rd Tilson, Immune cells and molecular mediators in the pathogenesis of the abdominal aortic aneurysm. Cardiol Rev 17 (2009), 201-210.
[24] C. Saracini, P. Bolli, E. Sticchi, G. Pratesi, R. Pulli, F. Sofi, C. Pratesi, G.F. Gensini, R. Abbate, B. Giusti, Polymorphisms of genes involved in extracellular matrix remodeling and abdominal aortic aneurysm. J Vasc Surg 55 (2012), 171-179.

[25] M. Seifi, S. Fallah, M. Firoozrai, Influence of genetic polymorphism in matrix metalloproteinase- 3 on extent of coronary atherosclerosis and risk of coronary artery stenosis. Arch Med Res 40 (2009), 600-604.

[26] K. Stankunas, C. Shang, K.Y. Twu, S.C. Kao, N.AY. Jenkins, N.G. Copeland, M. Sanyal, L. Selleri, M.L. Cleary, C.P. Chang, Pbx/Meis deficiencies demonstrate multigenetic origins of congenital heart disease. Circ Res 103 (2008), 702709.

[27] G.K. Sukhova, U. Schönbeck, E. Rabkin, F.J. Schoen, A.R. Poole, R.C. Billinghurst, P. Libby, Evidence for increased collagenolysis by interstitial collagenases-1 and -3 in vulnerable human atheromatous plaques. Circulation 99 (1999), 25032509.

[28] C. Tarín, M. Gomez, E. Calvo, J.A. López, C. Zaragoza, Endothelial nitric oxide deficiency reduces MMP-13-mediated cleavage of ICAM-1 in vascular endothelium: a role in atherosclerosis. Arterioscler Thromb Vasc Biol 29 (2009), 2732 .

[29] A. Vasku, M. Goldbergová, L. Izakovicová Hollá, L. Sisková, L. Groch, M. Beránek, S. Tschöplová, V. Znojil, J. Vácha, A haplotype constituted of four MMP-2 promoter polymorphisms $(-1575 \mathrm{G} / \mathrm{A},-1306 \mathrm{C} / \mathrm{T},-790 \mathrm{~T} / \mathrm{G}$ and $-735 \mathrm{C} / \mathrm{T})$ is associated with coronary triple-vessel disease, Matrix Biol 22 (2004), 585-591.

[30] Y. Yu, T. Koike, S. Kitajima, E. Liu, M. Morimoto, M. Shiomi, K. Hatakeyama, Y. Asada, K.Y. Wang, Y. Sasaguri, T. Watanabe, J. Fan, Temporal and quantitative analysis of expression of metalloproteinases (MMPs) and their endogenous inhibitors in atherosclerotic lesions. Histol Histopathol 23 (2008), 1503-1516.

[31] B. Zhang, S. Ye, S.M. Herrmann, P. Eriksson, M. de Maat, A. Evans, D. Arveiler, G. Luc, F. Cambien, A. Hamsten, H. Watkins, A.M. Henney, Functional polymorphism in the regulatory region of gelatinase $\mathrm{B}$ gene in relation to severity of coronary atherosclerosis. Circulation 99 (1999), 1788-1794. 


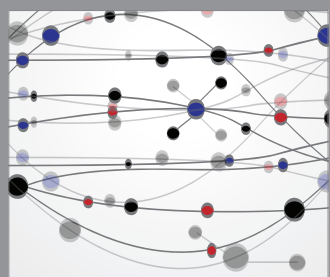

The Scientific World Journal
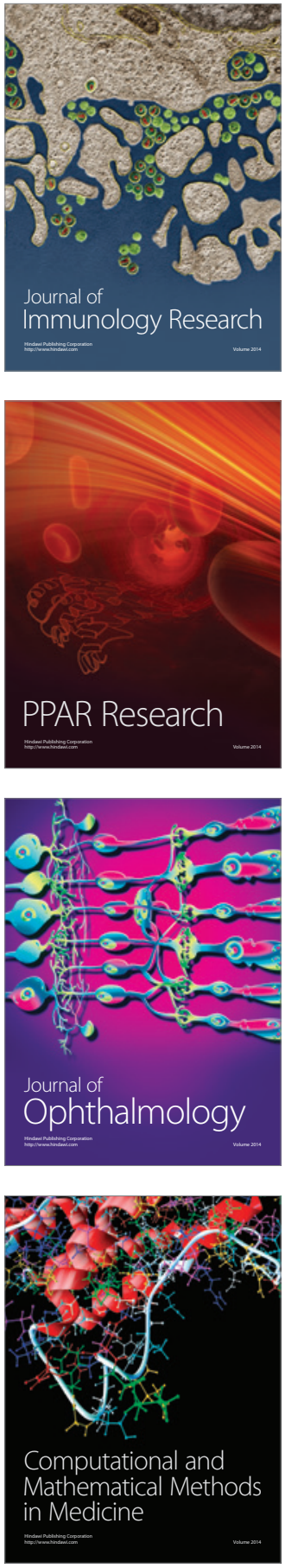

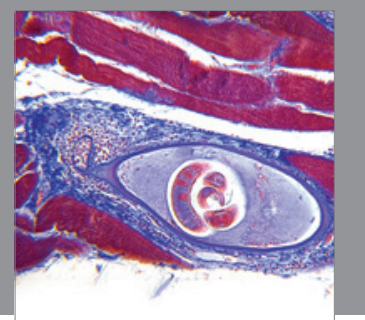

Gastroenterology

Research and Practice
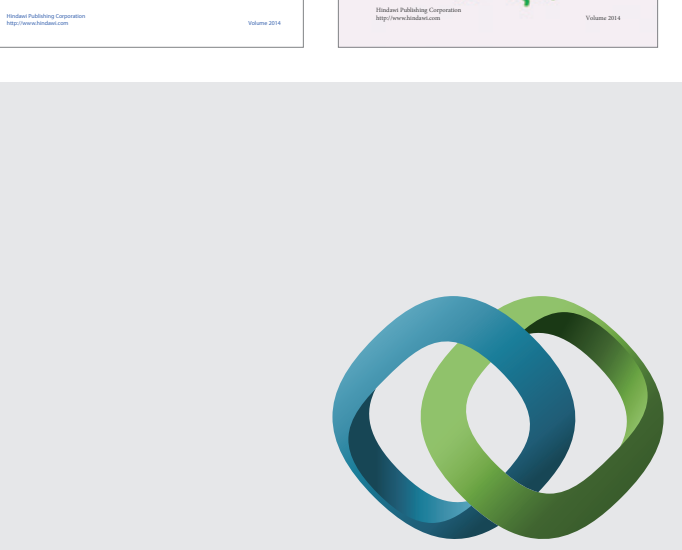

\section{Hindawi}

Submit your manuscripts at

http://www.hindawi.com
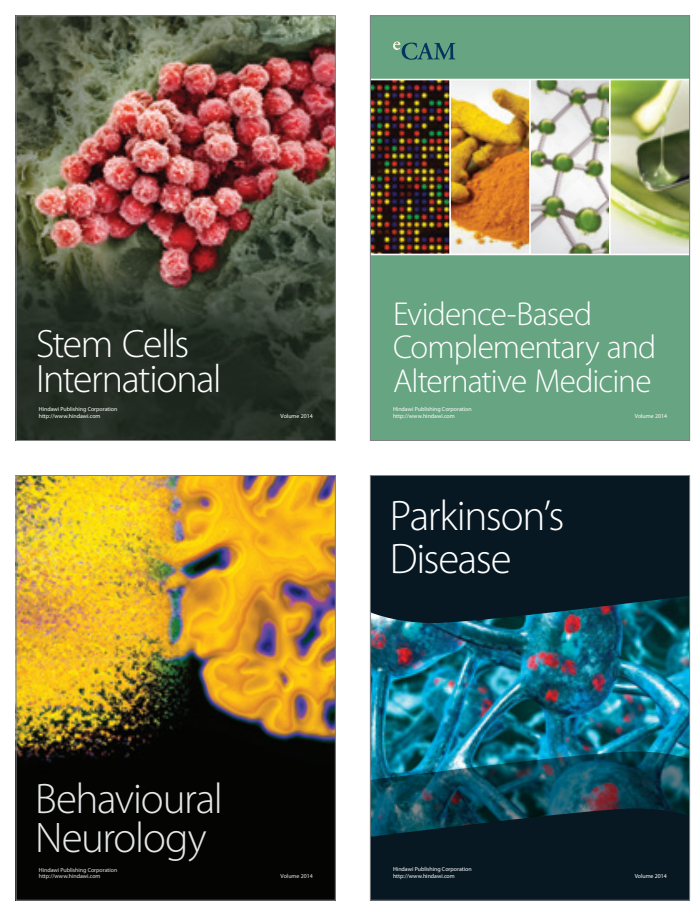

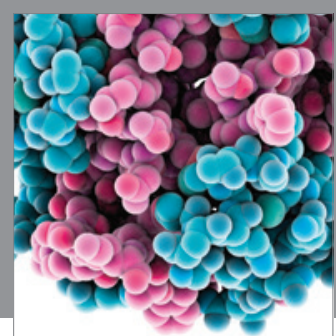

Journal of
Diabetes Research

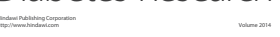

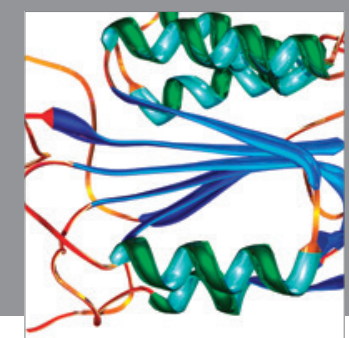

Disease Markers
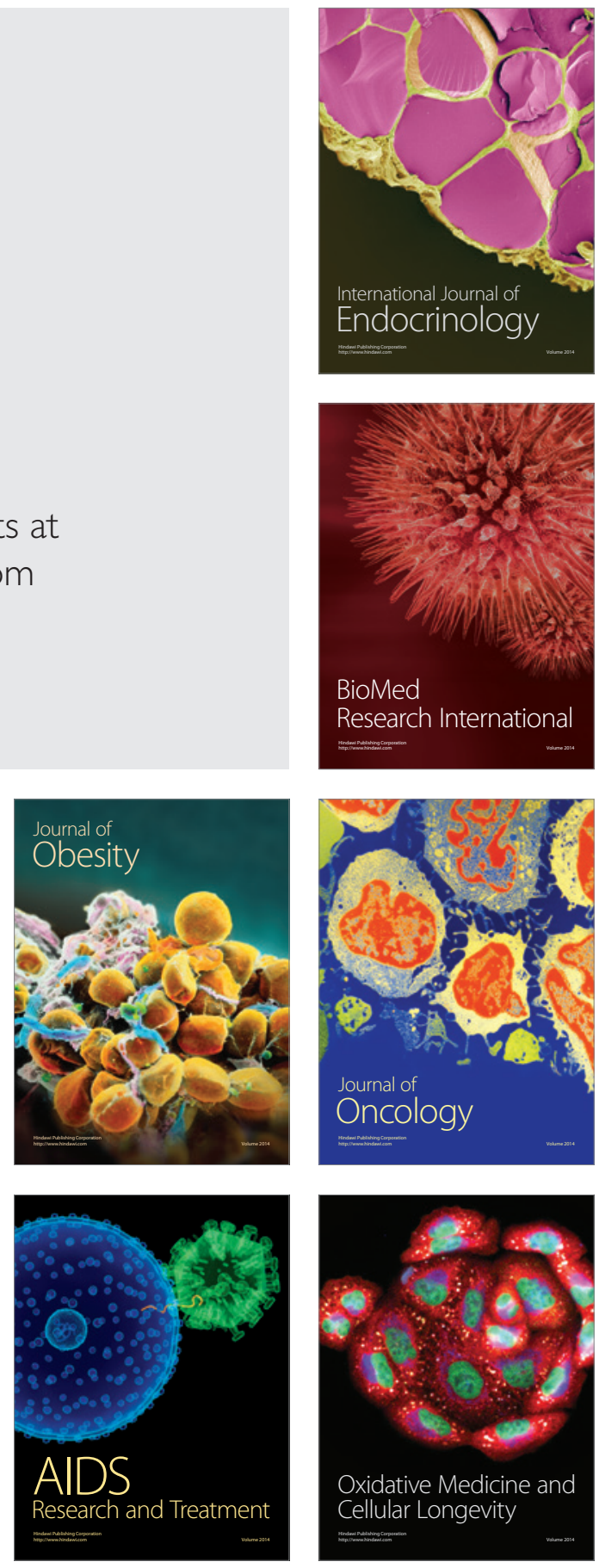